Supporting information

\title{
Determining the Absolute Concentration of Nanoparticles Without Calibration Factor by Visualizing the Dynamic Processes of Interfacial Adsorption
}

Xiang $\mathrm{Wo}^{\dagger}$, Zhimin $\mathrm{Li}^{\dagger}$, Yingyan Jiang ${ }^{\dagger}$, Minghe $\mathrm{Li}^{\dagger}$, Yu-wen $\mathrm{Su}^{\ddagger}$, Wei Wang ${ }^{* \dagger}$, Nongjian Tao ${ }^{\dagger}$

† State Key Laboratory of Analytical Chemistry for Life Science, School of Chemistry and Chemical Engineering, Nanjing University, Nanjing 210093, China

¥ School of Pharmacy, Nanjing Medical University, Nanjing 211166, China

Corresponding author email: wei.wang@nju.edu.cn (W. W.)

\section{Deduction of hitting frequency equation (Eq. 1)}

The presence of an adsorption layer in a solution-substrate interface resulted in a classical semi-infinite diffusion system. Based on Fick's second law of diffusion, the local concentration of nanoparticle is a function of time and location:

$$
\frac{\partial C(x, t)}{\partial t}=D \frac{\partial^{2} C(\mathrm{x}, \mathrm{t})}{\partial x}
$$

under following initial and boundary conditions:

$$
\begin{array}{ll}
C(x, 0)=C_{0} & \text { (initial condition) } \\
C(0, t)=0(t>0) & \text { (Hit-n-Stay creates zero-concentration layer) } \\
\lim _{x \rightarrow \infty} C(x, t)=C_{0} & \text { (infinite diffusion) }
\end{array}
$$

where $C(x, t)$ is the local concentration of nanoparticles, $x$ is the distance towards the adsorption layer, and $D$ is the diffusion coefficient.

Hitting frequency was determined by the concentration gradient at the interface:

$$
f(t)=D\left[\frac{\partial C(x, t)}{\partial x}\right]_{x=0}
$$

The combination of Eqs. (1) and (2) gives an analytical expression of hitting frequency as below:

$$
f(t)=\frac{N_{A} A D^{1 / 2} C_{0}}{\pi^{1 / 2}} t^{-1 / 2}
$$

where $f(t)$ is the hitting frequency as a function of time, $N_{\mathrm{A}}$ is Avogadro's constant, $A$ is the area of the observation region, $D$ is the diffusion coefficient of nanoparticles, and $C_{0}$ is the number concentration of nanoparticles. 


\section{Characterization of polystyrene nanoparticles}

The polystyrene nanoparticles used in the present work were characterized by transmission electron microscopy (TEM) and dynamic light scattering (DLS) to access the morphology and size distribution. It was found that polystyrene nanoparticles were nicely spherical and the size distribution was narrow and uniform, with an average radius of $107 \mathrm{~nm}$ and a standard deviation of $4 \mathrm{~nm}$. The TEM radius was slightly smaller than DLS radius of $110 \mathrm{~nm}$, which was quite common for polymer nanoparticles.

(a)

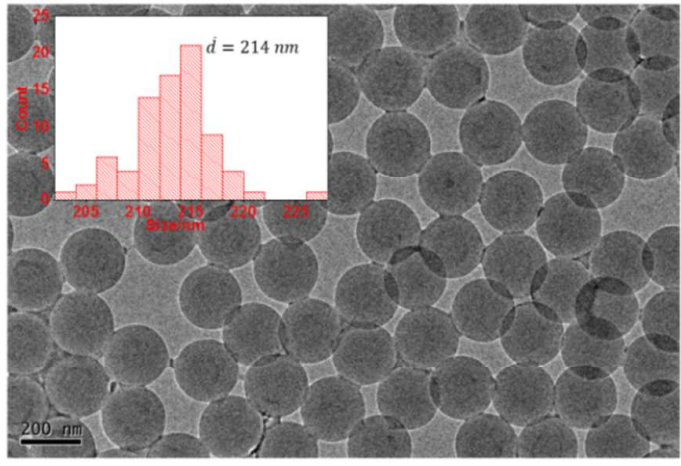

(b)

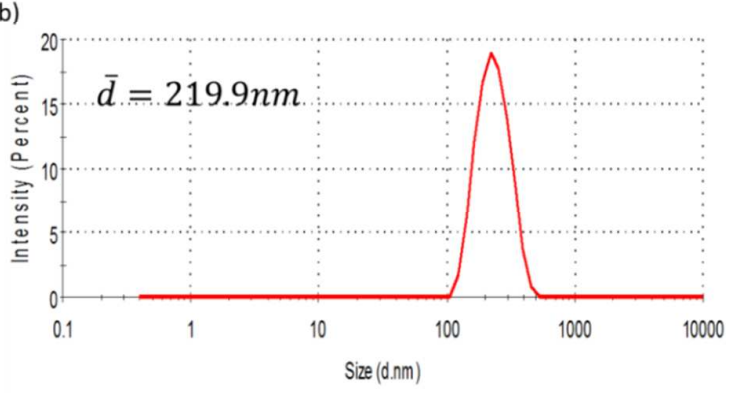

Figure S1. (a) TEM and (b) DLS results of polystyrene nanoparticles.

\section{Distance constant for nanoparticles with different size and refractive index}

In order to examine the distance constant for nanoparticles with different size and refractive index, COMSOL simulation were performed while adjusting the value of size and refractive index, respectively. Despite of the different electric field values, similar distance constants were obtained under three models, demonstrating the independence of distance constant with the size and refractive index of nanoparticles.
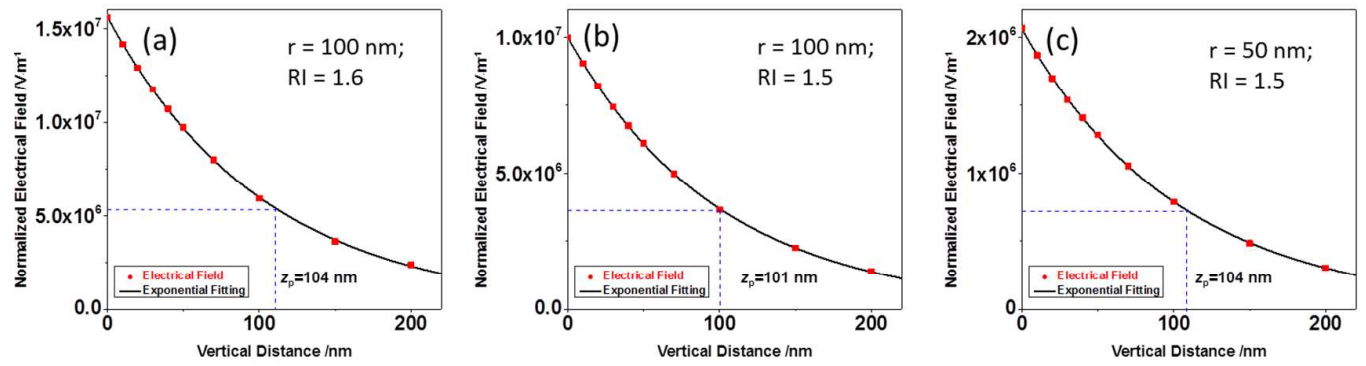

Figure S2. Distance constant resolved from the COMSOL simulation for nanoparticles with different radius $(r)$ and refractive index (RI). (a) $r=100 \mathrm{~nm}, \mathrm{RI}=1.6$; (b) $r=100 \mathrm{~nm}, \mathrm{RI}=1.5 ;$ (c) $r=50 \mathrm{~nm}, \mathrm{RI}=1.5$. 


\section{AFM images}

SPR microscopy does not put a strong requirement on the roughness of gold film because the characteristic propagation distance of surface plasmon polariton is around 5 microns. Typical roughness of gold film used in our lab is round $5 \mathrm{~nm}$ per $\mathrm{um}^{2}$, which can be easily achieved by commercial vacuum evaporators.

AFM image of the substrate after SPRM measurements exhibited an even distribution of nanoparticles, which is consistent with SPRM results.
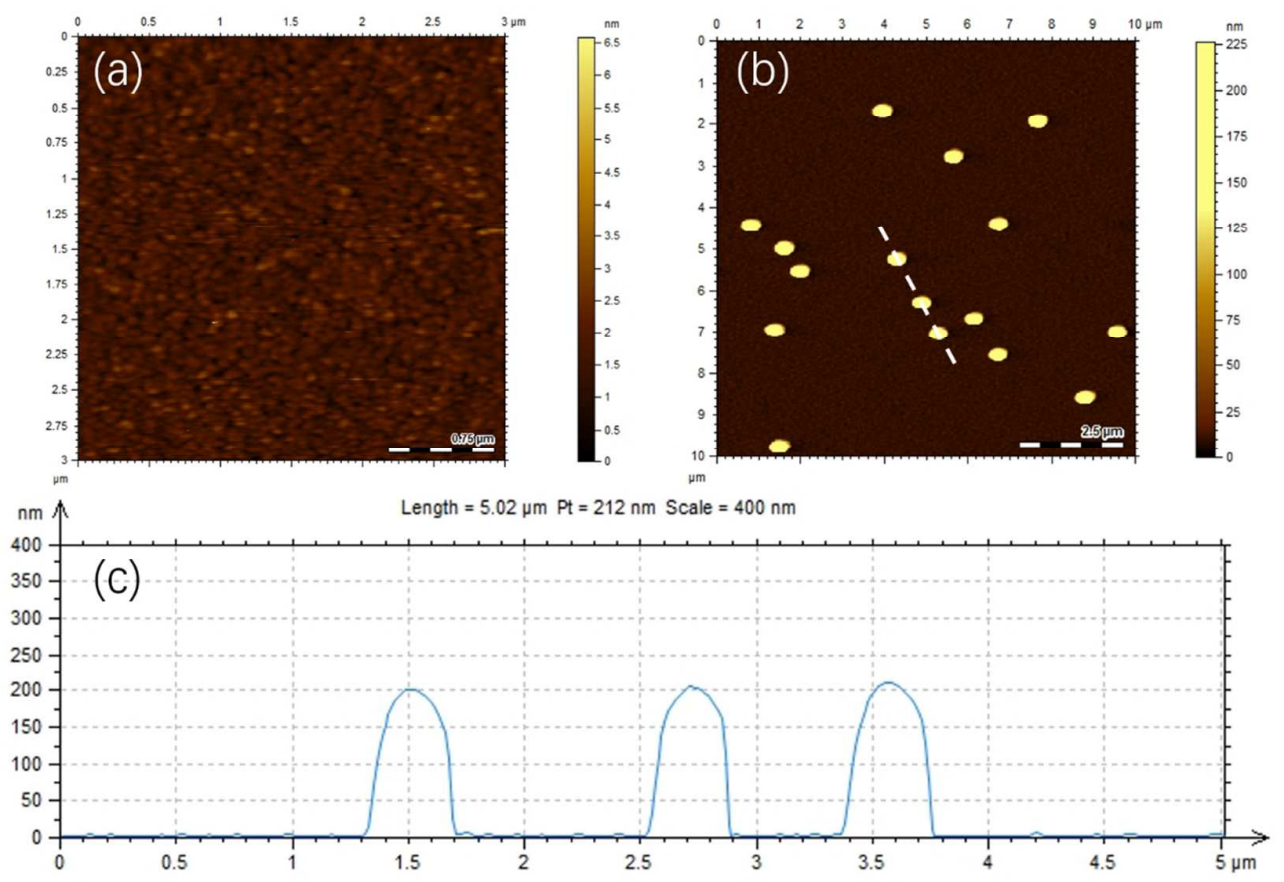

Figure S3. Typical AFM image of (a) blank gold substrate and (b) gold substrate after the adsorption of polystyrene NPs. (c) The height profile of three polystyrene NPs as indicated by the white dash line in (b).

\section{Electrochemical Impedance Spectroscopy (EIS) images}

EIS measurements were carried out in solution containing $0.1 \mathrm{M} \mathrm{KCl}, 1 \mathrm{mM} \mathrm{Fe}(\mathrm{CN})_{6}{ }^{4-}$ and $1 \mathrm{mM} \mathrm{Fe}(\mathrm{CN})_{6}^{3-}$. The greatly increased electrochemical impedance of PEG modified gold film demonstrated the successful assembly of PEG self-assembled layer.
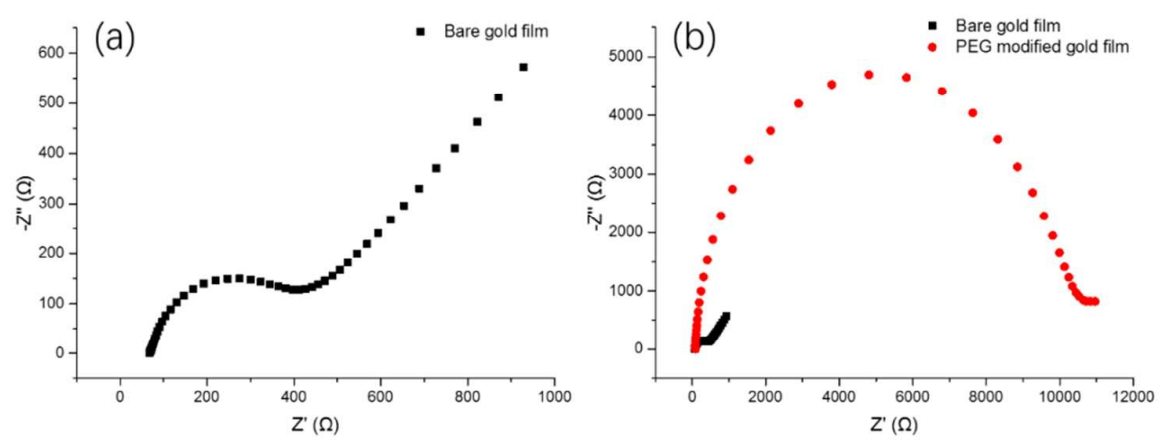

Figure S4. Typical EIS spectrum measured on (a) bare gold film (black curve) and (b) PEG modified gold film (red curve). 